\title{
Plantio Misto de Eucalyptus spp. com Leguminosas Arbóreas Fixadoras de Nitrogênio
}

\author{
Márcio Viera ${ }^{1}$, Mauro Valdir Schumacher ${ }^{2}$, Edenilson Liberalesso ${ }^{3}$, \\ Marcos Vinícius Winckler Caldeira ${ }^{4}$, Luciano Farinha Watzlawick ${ }^{5}$
}

${ }^{1}$ Departamento Multidisciplinar, Universidade Federal de Santa Maria - UFSM, Silveira Martins/RS, Brasil ${ }^{2}$ Departamento de Ciências Florestais - DCFL, Universidade Federal de Santa Maria - UFSM, Santa Maria/RS, Brasil ${ }^{3}$ Engenheiro Florestal, Empresa Duratex S/A, Taquari/RS, Brasil

${ }^{4}$ Universidade Federal do Espírito Santo - UFES, Alegre/ES, Brasil

${ }^{5}$ Universidade Estadual do Centro-Oeste - UNICENTRO, Guarapuava/PR, Brasil

\begin{abstract}
RESUMO
A revisão relata os principais benefícios do plantio misto de eucalipto com leguminosas arbóreas fixadoras de nitrogênio atmosférico. Buscou-se analisar as interações ecológicas das espécies envolvidas no sistema, a arquitetura espacial utilizada e o possível aumento de produtividade do sistema consorciado. Com base nisso, observa-se que o cultivo misto de eucaliptos com leguminosas arbóreas fixadoras de nitrogênio atmosférico só deve ser realizado em locais onde os processos e as interações entre espécies aumentarão o crescimento, a produção de biomassa ou a qualidade do produto final desejado. De forma geral, os estudos indicam que o interplantio de leguminosas arbóreas fixadoras de nitrogênio proporciona incremento na produtividade das plantas de eucalipto. Contudo, devem-se analisar as interações dos plantios mistos até a sua idade de rotação, para que, com isso, possam ser verificados os possíveis efeitos da fixação biológica de nitrogênio atmosférico pela leguminosa na produtividade do eucalipto.
\end{abstract}

Palavras-chave: consórcio de espécies florestais, interações ecológicas, produtividade florestal.

\section{Mixed Plantation of Eucalyptus with Nitrogen-fixing Legume Trees}

\begin{abstract}
In this paper, we aimed to review the main benefits of the mixed plantation of eucalyptus with nitrogen-fixing legume trees. To this end, the ecological interactions of the species involved in the system was analyzed, as well as the space architecture used and the possible increase in productivity caused by the intercropping. On this basis, it was possible to observe that the mixed cropping of eucalyptus with nitrogen-fixing legume trees should be implemented only in locations where the processes and interactions between species will increase the growth, biomass production or quality of desired product. In general, studies indicate that mixed plantation provides an increase in productivity in eucalyptus stand. However, it is important to analyze the interactions of mixed stand until the age of rotation, so that the possible effects of atmospheric nitrogen fixation by legume trees on the productivity of eucalyptus can be verified.
\end{abstract}

Keywords: forestry intercropping, ecological interactions, forestry productivity. 


\section{INTRODUÇÃO}

As plantações florestais estão em constante crescimento, sendo que hoje o cultivo de eucalipto ocupa uma área em torno de sete milhões de hectares no mundo, dos quais mais da metade está plantada no Brasil (FAO, 2009), representando mais de 50\% da área com florestas exóticas implantadas no País (ABRAF, 2009). O eucalipto está entre as principais fontes de matéria-prima para a produção de celulose e de carvão vegetal para siderurgia e fábricas de cimento, bem como para serrarias, postes, óleos essenciais, entre outras finalidades (Lima, 1996). A intensa utilização decorre do seu crescimento satisfatório em solos de baixa fertilidade natural e da sua eficiência de utilização dos nutrientes (Reis et al., 1989). Segundo os autores, estes fatores contribuem para intensificar a implantação florestal, em locais antes sem tradição silvicultural para essas espécies.

No entanto, a expansão do cultivo de monoculturas pode trazer impactos negativos ao ambiente local em razão da falta de diversificação na produção rural, além da exploração ineficiente dos recursos do solo, quando comparada com a utilização diversificada de espécies (Erskine et al., 2006). Em contrapartida, diversos trabalhos demonstram que a implementação de plantios mistos pode fornecer benefícios por meio de bens e serviços ambientais, como proteção e conservação da biodiversidade, e recuperação de áreas degradadas (Parrota, 1999; Forrester et al., 2006c; Kelty, 2006; Piotto, 2008).

Além desses benefícios, plantios mistos de eucalipto e leguminosas arbóreas fixadoras de nitrogênio atmosférico resultam em aumento na produtividade das espécies envolvidas. Esse aumento foi verificado em Eucalyptus globulus com Acacia mearnsii (Khanna, 1997; Bauhus et al., 2000; Forrester et al., 2004, 2005, 2006a); Eucalyptus saligna com Albizia falcataria (Binkley et al., 2000); Eucalyptus saligna com Falcataria moluccana (Binkley et al., 2003); Eucalyptus grandis com Acacia mangium (Laclau et al., 2008); eucalipto com leguminosas arbóreas nativas (Coelho, 2006), e Eucalyptus camaldulensis com Acacia mangium (Schiavo, 2005).

Várias questões ainda necessitam ser avaliadas, como: por quanto tempo a leguminosa arbórea fixará nitrogênio $(\mathrm{N})$ nos povoamentos mistos; se as intervenções silviculturais - tais como desrama, desbaste e fertilização - podem influenciar a dinâmica de fixação de nitrogênio conforme a idade do povoamento; se aumentos de produção resultarão em aumentos na produtividade total - acima e abaixo do solo (Forrester et al., 2005), além de ser difícil predizer a combinação de espécies e sítios que poderiam apresentar benefícios (Forrester et al., 2006b). Dessa forma, essa revisão visa a demonstrar quais são os principais benefícios do plantio misto de eucalipto com leguminosas arbóreas fixadoras de nitrogênio atmosférico.

\section{INTERAÇÕES E PRODUTIVIDADE DAS ESPÉCIES ENVOLVIDAS EM CULTIVO MISTO}

\subsection{Interações ecológicas entre as espécies em cultivo misto}

Quando realizado o plantio consorciado de espécies florestais, as espécies envolvidas podem ser submetidas a interações ecológicas. Estas interações podem advir da competição, da redução competitiva ou da facilitação (Vandermeer, 1989). Segundo o autor, a competição ocorre quando dois ou mais indivíduos ou populações interagem de modo que pelo menos um exerça um efeito negativo sobre o outro (redução do crescimento ou mortalidade).

A redução competitiva decorre de uma menor competição interespecífica nos cultivos mistos do que a competição intraespecífica nas monoculturas (Kelty \& Cameron, 1995). Segundo os autores, esse tipo de interação pode ocorrer em função de um uso mais eficiente dos recursos inerentes do sítio, como luz, água ou nutrientes. Entretanto, a facilitação ocorre quando uma espécie interage com a outra provocando efeito positivo na produtividade (Vandermeer, 1989); por exemplo, quando uma espécie que fixa nitrogênio aumenta o crescimento da outra espécie que não é fixadora de nitrogênio, em virtude do aumento da disponibilidade do nutriente no sistema (Forrester et al., 2005).

Quando as interações positivas são dominantes (facilitação ou redução competitiva), povoamentos consorciados serão mais produtivos do que 
monoculturas (Forrester et al., 2004). Os autores mencionam que, se a competição interespecífica for maior do que estas interações positivas, os consórcios serão menos produtivos. É difícil prever o resultado das diversas interações em sistemas mistos, assim como a natureza dessas interações pode mudar o desenvolvimento das espécies envolvidas (Forrester et al., 2004). Por isso, segundo os autores, a obtenção de informações de espécies bem sucedidas em plantios mistos, de proporções adequadas de cada espécie e de locais adequados para as combinações são difíceis de serem obtidas em curto prazo de tempo.

Predomínio de interações positivas (facilitação e redução competitiva) sobre as negativas (competitividade) foi verificado por Forrester et al. (2005) num plantio misto de Eucalyptus globulus e Acacia mearnsii na Austrália, evidenciado por maior crescimento em altura e diâmetro, incremento em biomassa acima do solo e volume de madeira nos tratamentos com misturas das espécies. Esse padrão demonstra o benefício de seleção de espécies capazes de fixar uma quantidade significativa de $\mathrm{N}$ e tornálo prontamente disponível com a decomposição da serapilheira e das raízes finas.

\subsection{Arquitetura espacial dos plantios mistos}

Informações de espécies bem sucedidas em arranjos mistos, proporções adequadas de cada espécie e locais adequados para as combinações são difíceis de serem obtidas antes do final de uma rotação. Parrotta (1999) relata que existem poucos estudos analisando essa dinâmica de crescimento de plantações mistas de eucaliptos e espécies fixadoras de $\mathrm{N}$ até o final do ciclo (colheita florestal).

A combinação das espécies florestais em plantios mistos pode ser realizada das seguintes maneiras: i) as espécies podem ser interplantadas em linhas alternadas com diferentes proporções de cada uma; ii) as espécies podem ser interplantadas com alternância de espécies dentro da própria linha de plantio; iii) podem-se formar faixas de cultivo de leguminosas no interior do povoamento de eucalipto. Em qualquer uma dessas formas de cultivo misto, ainda não existe uma definição da melhor combinação de espécies a ser utilizadas (Parrotta, 1999).
Na Tabela 1, são apresentados alguns arranjos espaciais experimentais de plantios mistos de eucalipto com leguminosas arbóreas. Conforme os arranjos utilizados nos plantios mistos, o número de indivíduos por área pode ser aumentando em até 100\% (Bouillet et al., 2008; Laclau et al., 2008). Isso ocorre em áreas onde é realizado o plantio do eucalipto no espaçamento tradicional da região, objetivando a obtenção de determinado produto, e no interior desse povoamento é interplantada a leguminosa com o mesmo número de indivíduos, formando uma combinação de 100E:100A (por exemplo: $100 \%$ de eucalipto $+100 \%$ de Acacia mangium). Em outros casos, também se mantém o número tradicional de plantas de eucalipto e realiza-se o interplantio com mais $50 \%$ ou $25 \%$ de indivíduos de leguminosas arbóreas. Por fim, o mais comumente utilizado é a combinação de plantas das duas espécies perfazendo um número de indivíduos por área que não exceda ao correspondente no espaçamento tradicional do eucalipto.

Mesmo não havendo uma definição do melhor arranjo espacial de distribuição das espécies utilizadas no plantio misto, estas podem ser manejadas em uma série de sistemas silviculturais (Forrester et al., 2004), tais como: i) a leguminosa pode ser desbastada para liberação do eucalipto e vendida como uma fonte de rendimento antecipado; ii) quando o desbaste não é economicamente viável, a espécie leguminosa pode ser mantida no povoamento junto com o eucalipto, o qual se tornaria dominante (nesse caso, a leguminosa continuaria a fixar $\mathrm{N}_{2}$ ); iii) ambas as espécies poderiam ser desbastadas de modo que os indivíduos de eucaliptos com melhor crescimento sejam mantidos com algumas leguminosas.

\subsection{Efeito na produtividade das espécies em cultivo misto}

Numerosos estudos na literatura relatam que o plantio misto de eucalipto e espécies arbóreas fixadoras de nitrogênio aumenta a produtividade das espécies envolvidas, estando tal fato atrelado às interações que ocorrem entre as espécies (Khanna, 1997; Binkley et al., 2000; Bauhus et al., 2000; Forrester et al., 2004, 2006a; Coelho et al., 2007; Laclau et al., 2008; Bouillet et al., 2008). No entanto, existem outros estudos nos quais se observou pouca 
Tabela 1. Arranjo espacial de plantios mistos com eucaliptos e leguminosas arbóreas utilizados em diversos estudos.

Table 1. Mixed stands spatial arrangement with eucalyptus and leguminous trees used in several studies.

\begin{tabular}{|c|c|c|c|c|}
\hline Fonte & Espécies & Localização & Espaçamento & Mistura (\%) \\
\hline $\begin{array}{l}\text { Bristow et al. } \\
\qquad(2006)\end{array}$ & $\begin{array}{l}\text { Eucalyptus pellita } \\
\text { Acacia peregrina }\end{array}$ & $\begin{array}{l}\text { Queensland - } \\
\text { Austrália }\end{array}$ & $5 \mathrm{~m} \times 2 \mathrm{~m}$ & $\begin{array}{c}\text { 100E; 75E:25A; 50E:50A; } \\
\text { 25E:75A; } 100 \mathrm{~A}\end{array}$ \\
\hline $\begin{array}{l}\text { Khanna (1997); } \\
\text { Forrester et al. } \\
\quad(2004)\end{array}$ & $\begin{array}{l}\text { Eucalyptus globulus } \\
\text { Acacia mearnsii }\end{array}$ & $\begin{array}{l}\text { Victoria - } \\
\text { Austrália }\end{array}$ & $\begin{array}{l}3,3 \mathrm{~m} \times 2 \mathrm{~m} \\
3,3 \mathrm{~m} \times 3 \mathrm{~m}\end{array}$ & $\begin{array}{c}\text { 100E; 75E:25A; 50E:50A; } \\
\text { 25E:75A; 100A }\end{array}$ \\
\hline $\begin{array}{l}\text { Forrester et cl. } \\
\qquad(2006 \mathrm{~b})\end{array}$ & $\begin{array}{l}\text { Eucalyptus globulus } \\
\text { Acacia mearnsii }\end{array}$ & $\begin{array}{l}\text { Victoria - } \\
\text { Austrália }\end{array}$ & $3,3 \mathrm{~m} \times 3 \mathrm{~m}$ & $\begin{array}{l}\text { 100E; 50E:50A; } \\
\text { 100A }\end{array}$ \\
\hline Parrotta (1999) & $\begin{array}{l}\text { Eucalyptus robusta } \\
\text { Leucaena leucocephala } \\
\text { Casuarina equisetifolia }\end{array}$ & $\begin{array}{l}\text { Toa Baja - } \\
\text { Porto Rico }\end{array}$ & $1 \mathrm{~m} \times 1 \mathrm{~m}$ & $\begin{array}{c}\text { 100E; 100L; 100C; } \\
\text { 50E:50L; 50E:50C; 50L:50C }\end{array}$ \\
\hline $\begin{array}{l}\text { Bouillet et al. } \\
\quad \text { (2008); } \\
\text { Laclau et al. } \\
\quad(2008)\end{array}$ & $\begin{array}{l}\text { Eucalyptus grandis } \\
\text { Acacia mangium }\end{array}$ & $\begin{array}{l}\text { São Paulo - } \\
\text { Brasil }\end{array}$ & $3 \mathrm{~m} \times 3 \mathrm{~m}$ & $\begin{array}{l}\text { 100E; 100A; 25A:100E; } \\
\text { 50A:100E; } \\
\text { 100A:100E; 50A:50E }\end{array}$ \\
\hline $\begin{array}{l}\text { Balieiro et al. } \\
\qquad(2007)\end{array}$ & $\begin{array}{c}\text { Eucalyptus grandis } \\
\text { Pseudosamanea guachapele }\end{array}$ & $\begin{array}{l}\text { Rio de Janeiro - } \\
\text { Brasil }\end{array}$ & $3 \mathrm{~m} \times 1 \mathrm{~m}$ & 100E; 100P; 50E:50P \\
\hline Binkley (2003) & $\begin{array}{c}\text { Eucalyptus saligna } \\
\text { Facaltaria moluccana }\end{array}$ & $\begin{array}{l}\text { Havai - } \\
\text { USA }\end{array}$ & $2 \mathrm{~m} \times 2 \mathrm{~m}$ & $\begin{array}{l}\text { 100E; 100F; 89E:11F; } \\
\text { 75E:25F; 67E:33F; } \\
\text { 50E:50F; 25E:75F }\end{array}$ \\
\hline $\begin{array}{l}\text { Mendonça et al. } \\
\text { (2008) }\end{array}$ & $\begin{array}{l}\text { E. camaldulensis; E. pellita; E. } \\
\text { tereticornes; E. robusta; Mimosa } \\
\text { caesalpiniaefolia }\end{array}$ & $\begin{array}{l}\text { Rio de Janeiro - } \\
\text { Brasil }\end{array}$ & $3 \mathrm{~m} \times 2 \mathrm{~m}$ & $\begin{array}{c}100 \mathrm{E}_{\mathrm{c}} ; 100 \mathrm{E}_{\mathrm{p}} ; 100 \mathrm{E}_{\mathrm{t}} ; 100 \mathrm{E}_{\mathrm{r}} \\
50 \mathrm{E}_{\mathrm{c}} ; 50 \mathrm{M} ; 50 \mathrm{E}_{\mathrm{p}}: 50 \mathrm{M} ; \\
50 \mathrm{E}_{\mathrm{t}}: 50 ; 50 \mathrm{E}_{\mathrm{r}}: 50 \mathrm{M}\end{array}$ \\
\hline $\begin{array}{l}\text { Vezzani et al. } \\
\qquad(2001)\end{array}$ & $\begin{array}{l}\text { Eucalyptus saligna } \\
\text { Acacia mearnsii }\end{array}$ & $\begin{array}{l}\text { Rio Grande do Sul - } \\
\text { Brasil }\end{array}$ & $4 \mathrm{~m} \times 1,5 \mathrm{~m}$ & $100 \mathrm{E} ; 100 \mathrm{~A} ; 50 \mathrm{E}: 50 \mathrm{~A}$ \\
\hline $\begin{array}{l}\text { Coelho et al. } \\
\text { (2007) }\end{array}$ & $\begin{array}{c}\text { Eucalyptus grandis; } \\
\text { Peltophorum dubium; } \\
\text { Inga sp.; Mimosa scabrella; } \\
\text { Acacia polyphylla; Mimosa } \\
\text { caesalpiniaefolia; Acacia } \\
\text { mangium }\end{array}$ & $\begin{array}{l}\text { São Paulo - } \\
\text { Brasil }\end{array}$ & $3 \mathrm{~m} \times 3 \mathrm{~m}$ & $\begin{array}{l}\text { 100E; 100E:50P; 100E:50I; } \\
\text { 100E:50MS; 100E:50AP; } \\
\text { 100E:50MC; 100E:50AM }\end{array}$ \\
\hline Kleinpaul (2008) & $\begin{array}{l}\text { Eucalyptus urograndis } \\
\text { Acacia mearnsii }\end{array}$ & $\begin{array}{l}\text { Rio Grande do Sul - } \\
\text { Brasil }\end{array}$ & $4 \mathrm{~m} \times 1,5 \mathrm{~m}$ & $\begin{array}{c}\text { 100E; 75E:25A; 50E:50A; } \\
\text { 25E:75A; 100A }\end{array}$ \\
\hline $\begin{array}{l}\text { Viera et al. } \\
\text { (2011) }\end{array}$ & $\begin{array}{c}\text { Eucalyptus urograndis } \\
\text { Acacia mearnsii }\end{array}$ & $\begin{array}{c}\text { Rio Grande do Sul - } \\
\text { Brasil }\end{array}$ & $4 \mathrm{~m} \times 1,5 \mathrm{~m}$ & $\begin{array}{c}\text { 100E; 75E:25A; 50E:50A; } \\
\text { 25E:75A; } 100 \mathrm{~A}\end{array}$ \\
\hline
\end{tabular}

$\mathrm{E}=$ Eucalyptus $; \mathrm{E}=$ E. camaldulensis $; \mathrm{E}_{\mathrm{p}}=$ E. pellita $; \mathrm{E}_{\mathrm{t}}=$ E. tereticornes $; \mathrm{E}=$ E. robusta $; \mathrm{A}=$ Acacia $; \mathrm{C}=$ Casuarina $; \mathrm{L}=$ Leucaena $;$ $\mathrm{F}=$ Falcataria $; \mathrm{M}=$ Mimosa $; \mathrm{P}=$ Pseudosamanea $; \mathrm{I}=$ Inga $; \mathrm{MS}=$ Mimosa scabrella $; \mathrm{AP}=$ Acacia polyphylla $; \mathrm{MC}=$ Mimosa caesalpiniaefolia; $\mathrm{AM}=$ Acacia mangium

ou nenhuma vantagem no crescimento dos eucaliptos (Jesus \& Dias, 1988; Debell et al., 1997; Viera et al., 2011), e até mesmo o prejuízo no crescimento da outra espécie florestal, as leguminosas (Parrotta, 1999; Hunt et al., 2006).

A predição da combinação de espécies e sítios que poderia apresentar esses benefícios ou restrições no crescimento ainda é difícil. Dessa forma, o plantio misto bem sucedido é aquele em que a produtividade das espécies envolvidas aumenta em relação ao seu monocultivo, reforçando a ideia de que esse aumento de produtividade em plantios mistos se deve principalmente à alta taxa de ciclagem de nutrientes. Por isso, é importante selecionar espécies fixadoras de nitrogênio, com fácil decomposição de serapilheira e alta taxa de ciclagem de nutrientes, assim como alta capacidade de fixação de $\mathrm{N}_{2}$ (Forrester et al., 2006b).

Uma vasta revisão de literatura sobre plantios mistos - realizada por Forrester et al. (2006b); Piotto (2008) e Richards et al. (2010) - revelou que, em geral, as misturas de espécies florestais são mais produtivas do que as monoculturas e que os plantios mistos podem ser fundamentais para satisfazer 
a necessidade econômica por meio de rotações sortidas e, ainda, fornecer benefícios ecológicos.

Por meio de análise da literatura, Richards et al. (2010) observaram que a concentração foliar de $\mathrm{N}$ aumentou significativamente para as espécies interplantadas com leguminosas arbóreas fixadoras de nitrogênio em relação ao cultivo em monocultura. Os autores sugerem que esse efeito decorre do aumento da taxa de fotossíntese e da eficiência do uso dos recursos do sítio florestal. Com isso, a utilização de espécies leguminosas fixadoras de nitrogênio aumentaria a quantidade deste elemento no sítio florestal. Forrester et al. (2007) observaram, aos dez anos de idade do povoamento em espaçamento de 3,3 $\mathrm{m} \times 3,0 \mathrm{~m}$, uma taxa média de fixação de nitrogênio atmosférico de 38 e $86 \mathrm{~kg} \mathrm{ha}^{-1} \mathrm{ano}^{-1}$, para a $A$. mearnsii em plantio misto (50E:50A) e em monocultivo, respectivamente. Outro fator importante na disponibilidade de nitrogênio no sistema é a sua entrada pela água da chuva que, segundo Balieiro et al. (2007), é intensificada pela espécie leguminosa, podendo chegar a $12,89 \mathrm{~kg}$ $\mathrm{ha}^{-1} \mathrm{ano}^{-1}\left(\mathrm{NH}_{4}\right)$ em consórcios de E. grandis e Pseudosamenea guachapele em espaçamento de $3,0 \mathrm{~m} \times 1,0 \mathrm{~m}$.

Esse aumento de $\mathrm{N}$ na biomassa refletiria, também, na quantidade disponível no solo, pois a serapilheira formada a partir das leguminosas possuirá maiores teores de nitrogênio, o que torna mais rápido o processo de decomposição dos resíduos vegetais, em função da maior disponibilidade de nitrogênio para a atividade microbiana (Vezzani, 1997; Forrester et al., 2006c). Além do mais, plantios consorciados podem melhorar a agregação do solo, aumentar o teor de matéria orgânica e promover condições ecofisiológicas favoráveis ao crescimento das árvores (Gama-Rodrigues, 1997; Mendonça et al., 2008).

\subsection{Crescimento em diâmetro das espécies em cultivo misto}

Em plantios monoespecíficos e mistos de Eucalyptus grandis e Acacia mangium (100E; 100A; 25A:100E; 50A:100E; 100A:100E; 50A:50E) no Brasil, com 29 meses de idade em espaçamento de $3,0 \mathrm{~m} \times 3,0 \mathrm{~m}$, Silva (2007) verificou que o Eucalyptus grandis no plantio misto não apresentou diminuição ou aumento no crescimento em área basal em relação ao seu monocultivo. Kleinpaul (2008) também não observou diferença no crescimento em diâmetro de colo, aos dez meses de idade, em monoculturas e plantios mistos de E. urograndis e Acacia mearnsii em espaçamento de 4,0 $\mathrm{m} \times 1,5 \mathrm{~m}$. Em estudo realizado na Austrália, Forrester et al. (2004) observaram que o crescimento inicial da acácia-negra em plantios mistos é maior do que o eucalipto em consórcio, em espaçamento de $3,3 \mathrm{~m} \times 2,0 \mathrm{~m}$. Os autores observaram que os diâmetros de Eucalyptus globulus foram significativamente maiores em consórcios do que em monoculturas, aos quatro anos de idade, sendo que essas diferenças aumentaram com o tempo, quando Forrester et al. (2010), analisando o povoamento aos 15 anos de idade, observaram que, no cultivo misto (50E:50A), o diâmetro à altura do peito $($ A. mearnsii $=14,1 \mathrm{~cm}$ e E. globulus $=15,0 \mathrm{~cm})$ foi significativamente superior ao monocultivo do eucalipto $(11,8 \mathrm{~cm})$ e da acácia-negra $(11,7 \mathrm{~cm})$.

Em estudo realizado no Brasil com povoamentos monoespecíficos e mistos, em espaçamento de 4,0 m $\times 1,5 \mathrm{~m}$, Viera et al. (2011) observaram que, para o Eucalyptus urograndis e principalmente para a Acacia mearnsii, há diminuição da diferença entre o número de árvores por hectare com diâmetros superiores à proporção que aumenta a presença da outra espécie (Figura 1). Para efeito comparativo, os autores definiram como árvore de diâmetro superior aquela que tinha diâmetro à altura do peito superior à média mais um desvio-padrão. Para o Eucalyptus urograndis, o diâmetro é superior a $8,4 \mathrm{~cm}$ e para a Acacia mearnsii, superior a $8,7 \mathrm{~cm}$, aos 18 meses de idade.

Para a Acacia mearnsii, Forrester et al. (2004) observaram que ocorreu aumento significativo no número de árvores com diâmetros maiores com o aumento da proporção de Eucalyptus globulus; já para o Eucalyptus globulus, não houve aumento significativo no número de árvores com diâmetro superior com o aumento da proporção de Acacia mearnsii aos 11 anos de idade, em espaçamento de $3,3 \mathrm{~m} \times 2,0 \mathrm{~m}$. Dessa forma, plantios mistos apresentam maior proporção de árvores em classes de diâmetro superior do que as monoculturas. Esse padrão indica que a concorrência interespecífica na mistura foi menor do que na monocultura, levando a um maior crescimento das árvores em misturas, 

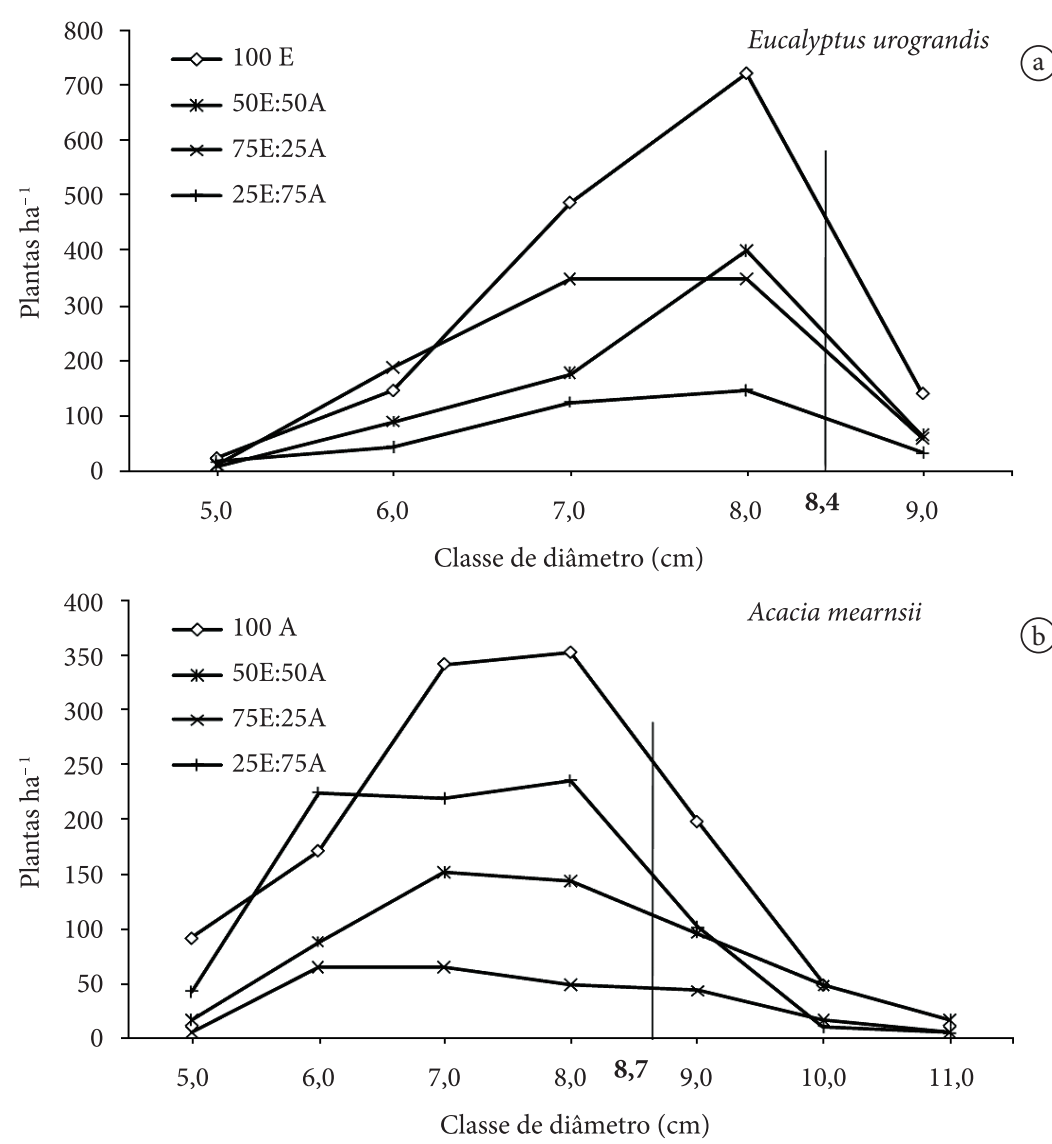

Figura 1. Distribuição diamétrica das árvores em plantios monoespecíficos e mistos de Eucalyptus urograndis e Acacia mearnsii aos 18 meses de idade (Viera et al., 2011). A = Acacia mearnsii; $\mathrm{E}=$ Eucalyptus urograndis.

Figure 1. Tree diameter distribution in monoespecific and mixed Eucalyptus urograndis and Acacia mearnsii stand 18 years-old (Viera et al., 2011). Where: $\mathrm{A}=$ Acacia mearnsii and $\mathrm{E}=$ Eucalyptus urograndis.

ocasionado pela maior facilitação e/ou redução competitiva pelos recursos do sítio (água, luz e nutrientes).

\subsection{Crescimento em altura das espécies em cultivo misto}

Alguns estudos, tais como de Vezzani et al. (2001), não encontraram diferença significativa no crescimento em diâmetro e altura de Eucalyptus saligna e Acacia mearnsii entre os sistemas de cultivo, aos 45 meses de idade, em um espaçamento de $4,0 \mathrm{~m} \times 1,5 \mathrm{~m}$. Os mesmos autores salientam que, embora o eucalipto consorciado tenha sido favorecido pelo maior suprimento de nitrogênio da acácia-negra, essa maior disponibilidade não acarretou maior crescimento das plantas. Silva
(2007), avaliando plantios monoespecíficos e mistos de Eucalyptus grandis e Acacia mangium, com 29 meses de idade, também não verificou influência significativa do consórcio no crescimento em altura. O mesmo foi verificado por Kleinpaul (2008), aos dez meses de idade, em monoculturas e plantios mistos de E. urograndis e Acacia mearnsii.

Entretanto, Coelho (2006) observou que o crescimento em altura do Eucalyptus grandis nos diferentestratamentosconsorciadoscomleguminosas arbóreas em espaçamento de $3,0 \mathrm{~m} \times 3,0 \mathrm{~m}$ apresentou diferença apenas aos 24 meses de idade. O eucalipto consorciado com a Mimosa scabrella cresceu menos; já as demais espécies leguminosas (Acacia mangium, Inga sp., Peltophorum dubium, Acacia polyphylla e Mimosa caesalpiniaefolia) 
não interferiram no crescimento do eucalipto. Forrester et al. (2004) também puderam constatar a influência dos consórcios apenas a partir do segundo ano de idade do plantio, quando tanto a altura do Eucalyptus globulus como a da Acacia mearnsii aumentaram significativamente com a presença da outra espécie.

Em razão de o eucalipto ser intolerante à sombra, devem-se escolher espécies fixadoras de nitrogênio com menor crescimento em altura e que sejam tolerantes a certo grau de sombreamento. Forrester et al. (2004) observaram que a Acacia mearnsii apresentou maior crescimento em altura no consórcio com o Eucalyptus globulus do que com plantas da própria espécie, principalmente a partir dos cinco anos de idade do povoamento. Verificaram também que a redução competitiva por luminosidade decorre da formação de um dossel estratificado na fase final de desenvolvimento.

\subsection{Produção de biomassa das espécies em cultivo misto}

No estudo realizado por Viera \& Schumacher (2011), avaliando a alocação de biomassa total acima do solo pelo Eucalyptus urograndis e pela Acacia mearnsii, tanto em monocultivo como em plantio misto, observou-se que não ocorreram diferenças significativas até os 18 meses de idade do povoamento. Quando levada em consideração a compartimentação da biomassa, os autores verificaram uma atenuação significativa na produção de biomassa de copa (folhas e galhos) da Acacia mearnsii, quando em consórcio com Eucalyptus urograndis (50E:50A), em relação ao seu cultivo monoespecífico. Note-se que o mesmo não ocorreu para o eucalipto. Dessa forma, pode-se observar a interação competitiva interespecífica entre o eucalipto e a acácia-negra, ocasionando redução da formação de biomassa de copa da leguminosa. Esse padrão pode estar correlacionado com a diminuição da disponibilidade de luz ocasionada por maior ocupação do espaço pela copa do Eucalyptus urograndis em relação à da acácia-negra, provocando o início de uma possível estratificação do dossel.

Segundo Forrester et al. (2004), o crescimento inicial (primeiros anos de idade) da acácia-negra em plantios mistos é maior do que o do eucalipto em cultivos estabelecidos na Austrália. Os autores observaram que o incremento médio anual em biomassa da acácia-negra era maior do que o do eucalipto até aos 4 anos de idade, quando ocorreu inversão do crescimento, ou seja, o incremento da acácia-negra começou a reduzir e o do eucalipto a aumentar, até que fosse atingido um pico máximo aos 11 anos de idade. O mesmo padrão foi verificado por Coelho et al. (2007) que, estudando a interação entre plantios mistos de Eucalyptus grandis e leguminosas arbóreas, observaram que não houve diferenças significativas para a produção de biomassa de tronco e parte aérea (folha, galho e casca) do eucalipto solteiro ou consorciado.

A produção de biomassa acima do solo em monocultivos e plantio misto de E. globulus e A. mearnsii na Austrália, aos 15 anos de idade, em espaçamento de 3,3 $\mathrm{m} \times 2,0 \mathrm{~m}$, foi significativamente superior no cultivo misto, com proporção de 50E:50A (123,2 $\mathrm{Mg} \mathrm{ha}^{-1}$, sendo: 58,3 $\mathrm{Mg} \mathrm{ha}^{-1}$ de E. globulus e $64,9 \mathrm{Mg} \mathrm{ha}^{-1}$ de A. mearnsii), do que no monocultivo do eucalipto $\left(66,6 \mathrm{Mg} \mathrm{ha}^{-1}\right)$ e da acácia-negra $(78,0$ $\mathrm{Mg} \mathrm{ha}^{-1}$ ) (Forrester et al., 2010).

\subsection{Crescimento radicular das espécies em cultivo misto}

O sistema radicular de plantios consorciados e puros de Eucalyptus globulus e Acacia mearnsii não demonstrou, segundo Bauhus et al. (2000), sinergismo para a biomassa e para o comprimento de raízes finas entre as duas espécies no consórcio, indicando que, com isso, a acácia poderia ter forte competição por recursos do solo com a outra espécie consorciada. Não se pode estabelecer se o aumento da densidade de raízes finas de uma espécie em plantio consorciado ou das duas espécies é resultado de uma maior capacidade competitiva de uma delas ou se é resultado da redução de algum recurso (Coelho, 2006). Silva (2007) observou elevada exploração radicular do Eucalyptus grandis induzindo as raízes de Acacia mangium a crescer próximas à árvore nas camadas em que a pressão competitiva era inferior.

No estudo realizado por Viera (2010), não foram observadas interações entre os sistemas radiculares do eucalipto e da acácia-negra até os 18 meses de idade em espaçamento de $4,0 \mathrm{~m} \times 1,5 \mathrm{~m}$, visto que as plantas em cultivo misto não apresentaram 
diferenças significativas em crescimento de raízes finas em relação ao seu cultivo monoespecífico. $\mathrm{O}$ autor também verificou que o crescimento do sistema radicular até os oito meses de idade, tanto para o plantio em monocultura como para o plantio misto, atingiu uma ocupação em torno de $120 \mathrm{~cm}$ de distância do tronco das árvores; já aos 18 meses de idade, o sistema radicular das espécies florestais ocupava toda a área útil do sistema. E, nessa idade, a densidade de raízes finas foi superior no plantio monoespecífico de Acacia mearnsii em relação ao povoamento monoespecífico e misto de Eucalyptus urograndis. Segundo o autor, o conhecimento sobre o crescimento das raízes finas e a configuração do sistema radicular durante a fase inicial de desenvolvimento dos povoamentos pode ajudar nas decisões de práticas em que a silvicultura intensiva ou a agrossilvicultura é utilizada e, portanto, assegurar o uso mais eficiente dos recursos do solo.

Em plantios consorciados de Eucalyptus grandis e leguminosas arbóreas, Coelho (2006) observou comportamento diferente ao verificado no estudo anterior, em que a densidade de raízes finas de todas as leguminosas foi sempre menor em relação ao eucalipto nas camadas superiores do solo, sugerindo forte competição intraespecífica nessa camada. Essa autora salienta que a diferença na distribuição de raízes entre as espécies florestais poderia resultar principalmente das diferenças de competitividade entre as espécies para obter recursos naturais do solo.

\section{CONSIDERAÇÕES FINAIS}

Os estudos indicam que o plantio misto com leguminosas arbóreas fixadoras de nitrogênio proporciona, em muitos casos, maior incremento na produtividade em povoamentos de eucaliptos. Porém, devem-se analisar as interações dos plantios mistos até a sua idade de rotação, para que, com isso, possam-se verificar os possíveis efeitos da fixação biológica de nitrogênio atmosférico pela leguminosa na produtividade do eucalipto. Mesmo não havendo uma definição sobre qual o melhor arranjo de espécies a ser utilizado, ressalva-se que os plantios realizados com duas ou mais espécies florestais podem promover a diversificação dos produtos obtidos em uma mesma área pelo silvicultor.

\section{STATUS DA SUBMISSÃO}

Recebido: 02/10/2011

Aceito: $26 / 11 / 2012$

Publicado: 28/02/2013

\section{AUTOR(ES) PARA CORRESPONDÊNCIA}

\section{Márcio Viera}

Departamento Multidisciplinar, Unidade Descentralizada de Educação Superior - UDESSM, Universidade Federal de Santa Maria - (UFSM), Rua Francisco Guerino 407, Centro, CEP 97195-000, Silveira Martins, RS, Brasil e-mail: vieraflorestal@yahoo.com.br

\section{Mauro Valdir Schumacher}

Laboratório de Ecologia Florestal, Departamento de Ciências Florestais, Centro de Ciências Rurais, Universidade Federal de Santa Maria - UFSM, Av. Roraima, 1000, Cidade Universitária "Prof. Mariano da Rocha Filho”, Camobi, CEP 97105-900, Santa Maria, RS, Brasil e-mail: mvschumacher@gmail.com

\section{REFERÊNCIAS}

Associação Brasileira de Produtores de Florestas Plantadas - ABRAF. Anuário brasileiro da ABRAF: ano base 2008. Brasília: ABRAF; 2009.

Balieiro FC, Franco AA, Fontes RLF, Dias LE, Campello EFC, Faira SM. Evaluation of the throughfall and stemflow nutrient contents in mixed and pure plantations of Acacia mangium, Pseudosamenea guachapele and Eucalyptus grandis. Revista Árvore 2007; 31(2): 339-346. http://dx.doi.org/10.1590/S0100-67622007000200017

Bauhus J, Khanna PK, Menden N. Aboveground and belowground interactions in mixed plantations of Eucalyptus globulus and Acacia mearnsii. Canadian Journal Forestry Research 2000; 30(12): 1886-1894. http://dx.doi.org/10.1139/x00-141

Binkley D, Senock R, Bird S, Cole TG. Twenty years of standdevelopmentin pureand mixedstands of Eucalyptus saligna and nitrogen-fixing Facaltaria moluccana. Forest Ecology and Management 2003; 182(1-3): 93-102. http:// dx.doi.org/10.1016/S0378-1127(03)00028-8

Binkley D, Giardina C, Bashkin MA. Soil phosphorus pools and supply under the influence of Eucalyptus saligna and nitrogen-fixing Albizia falcataria. Forest 
Ecology and Management 2000; 128(3): 241-247. http:// dx.doi.org/10.1016/S0378-1127(99)00138-3

Bouillet P, Laclau JP, Gonçalves JLM, Moreira MZ, Trivelin PCO, Jourdan C et al. Mixed-species plantations of Acacia mangium and Eucalyptus grandis in Brazil 2: Nitrogen accumulation in the stands and biological $\mathrm{N}_{2}$ fixation. Forest Ecology and Management 2008; 255(12): 3918-3930. http://dx.doi. org/10.1016/j.foreco.2007.10.050

Bristow M, Vanclay JK, Brooks L, Hunt M. Growth and species interactions of Eucalyptus pellita in a mixed and monoculture plantation in the humid tropics of north Queensland. Forest Ecology and Management 2006; 233(2-3): 285-294. http://dx.doi. org/10.1016/j.foreco.2006.05.019

Coelho SRF. Crescimento e fixação de nitrogênio em plantios mistos de eucalipto e leguminosas arbóreas nativas [dissertação]. Piracicaba: Recursos Florestais, Universidade de São Paulo; 2006.

Coelho SRF, Gonçalves JLM, Mello SLM, Moreira RM, Silva EV, Laclau JP. Crescimento, nutrição e fixação biológica de nitrogênio em plantios mistos de eucalipto e leguminosas arbóreas. Pesquisa Agropecuária Brasileira 2007; 42(6): 759-768. http://dx.doi. org/10.1590/S0100-204X2007000600001

Debell DS, Cole TC, Whitesell CD. Growth, development and yield of pure and mixed stands of Eucalyptus and Albizia. Forest Science 1997; 43(2): 286-298.

Erskine PD, Lamb D, Bristow M. Tree species diversity and eco-system function: can tropical multi-species plantations generate greater productivity? Forest Ecology and Management 2006; 233(2-3): 205-210. http://dx.doi.org/10.1016/j.foreco.2006.05.013

Food and Agriculture Organization of the United Nations - FAO. State of the world's forests. Roma: FAO; 2009.

Forrester DI, Bauhus J, Cowie AL. On the success and failure of mixed species tree plantations: lessons learned from a model system of Eucalyptus globulus and Acacia mearnsii. Forest Ecology and Management 2005; 209(1-2): 147-155. http://dx.doi. org/10.1016/j.foreco.2005.01.012

Forrester DI, Bauhus J, Cowie AL. Carbon allocation in a mixed-species plantation of Eucalyptus globulus and Acacia mearnsii. Forest Ecology and Management 2006a; 233(2-3): 275-284. http://dx.doi. org/10.1016/j.foreco.2006.05.018

Forrester DI, Bauhus J, Cowie AL, Vanclay JK. Mixed-species plantations of Eucalyptus with nitrogen-fixiong trees: A review. Forest Ecology and Management 2006b; 233(2-3): 211-230. http://dx.doi. org/10.1016/j.foreco.2006.05.012
Forrester DI, Bauhus J, Khanna PK. Growth dynamics in a mixed-species plantation of Eucalyptus globulus and Acacia mearnsii. Forest Ecology and Management 2004; 193(1-2): 81-95. http://dx.doi. org/10.1016/j.foreco.2004.01.024

Forrester DI, Cowie AL, Bauhus J, Wood JT, Forrester RI. Efects of changing the supply of nitrogen and phosphorus on growth and interactions between Eucalyptus globulus and Acacia mearnsii in a pot trial. Plant and Soil 2006c; 280(1-2): 267-277. http://dx.doi. org/10.1007/s11104-005-3228-x

Forrester DI, Schortemeyer M, Stock WD, Bauhus J, Khanna PK, Cowie AL. Assessing nitrogen fixation in mixed and single-species plantations of Eucalyptus globulus and Acacia mearnsii. Tree Physiology 2007; 27(6): 1319-1328. PMid:17545131. http://dx.doi.org/10.1093/treephys/27.9.1319

Forrester DI, Theiveyanathan S, Collopy JJ, Marcaret NE. Enhanced water use efficiency in a mixed Eucalyptus globulus and Acacia mearnsii plantation. Forest Ecology and Management 2010; 259(9): 1761-1770. http:// dx.doi.org/10.1016/j.foreco.2009.07.036

Gama-Rodrigues AC. Ciclagem de nutrientes por espécies florestais em povoamentos puros e mistos, em solos tabuleiros da Bahia, Brasil [Tese]. Viçosa: Solos e Nutrição de Plantas, Universidade Federal de Viçosa; 1997.

Hunt MA, Battaglia M, Davidson NJ, Unwin GL. Competition between plantation Eucalyptus nitens and Acacia dealbata weeds in northeastern Tasmania. Forest Ecology and Management 2006; 233(2-3): 260-274. http://dx.doi.org/10.1016/j.foreco.2006.05.017

Jesus RM, Dias GBN. Eucalyptus/Leucaena mixture experiment - growth and yield. IPEF 1988; 39: 41-46.

Kelty MJ. The role of species mixtures in plantation forestry. Forest Ecology and Management 2006; 233(2-3): 195-204. http://dx.doi. org/10.1016/j.foreco.2006.05.011

Kelty MJ, Cameron IR. Plot designs for the analysis of species interactions in mixed stands. Commonwealth Forestry Review 1995; 74: 322-332.

Khanna PK. Comparison of growth and nutrition of young monocultures and mixed stands of Eucalyptus globulus and Acacia mearnsii. Forest Ecology and Management 1997; 94(1-3): 105-113. http://dx.doi. org/10.1016/S0378-1127(96)03971-0

Kleinpaul IS. Plantio misto de Eucalyptus urograndis e Acacia mearnsii em sistema agroflorestal [dissertação]. Santa Maria: Engenharia Florestal, Universidade Federal de Santa Maria; 2008.

Laclau JP, Bouillet JP, Gonçalves JLM, Silva EV, Jourdan C, Cunha MCS et al. Mixed-species plantations of Acacia mangium and Eucalyptus grandis 
in Brazil 1.Growth dynamics and aboveground net primary production. Forest Ecology and Management 2008; 255(12): 3905-3917. http://dx.doi. org/10.1016/j.foreco.2007.10.049

Lima WP. Impacto ambiental do eucalipto. 2nd ed. São Paulo: Ed. Universidade de São Paulo; 1996.

Mendonça AVR, Carneiro JGA, Barroso DG, Santiago RA, Freitas TAS, Souza JS. Desempenho de quatro espécies de Eucalyptus spp. em plantios puros e consorciados com sabiá (Mimosa caesalpiniaefolia Benth) em cava de extração de argila. Revista Árvore 2008; 32(3): 395-405. http://dx.doi.org/10.1590/ S0100-67622008000300002

Parrotta JA. Productivity, nutrient cycling, and succession in single- and mixed-species plantations of Casuarina equisetifolia, Eucalyptus robusta, and Leucaena leucocephala in Puerto Rico. Forest Ecology and Management 1999; 124(1): 45-77. http://dx.doi. org/10.1016/S0378-1127(99)00049-3

Piotto D. A meta-analysis comparing tree growth in monocultures and mixed plantations. Forest Ecology and Management 2008; 255(3-4): 781-786. http:// dx.doi.org/10.1016/j.foreco.2007.09.065

Reis GG, Reis MGF, Maestri M, Xavier A, Oliveira LM. Crescimento de Eucalyptus camaldulensis, E. grandis e E. cloeziana sob diferentes níveis de restrição radicular. Revista Árvore 1989; 13(1): 1-18.

Richards AE, Forrester DI, Bauhus J, SchererLorenzen M. The influence of mixed tree plantations on the nutrition of individual species: a review. Tree Physiology 2010; 30(9): 1192-1208. PMid:20472645. http://dx.doi.org/10.1093/treephys/tpq035
Schiavo JA. Revegetação de áreas degradadas pela extração de argila, com espécies micorrizadas de Acacia mangium, Sesbania virgata e Eucalyptus camaldulensis [tese]. Rio de Janeiro: Produção Vegetal, Universidade Estadual do Norte Fluminense; 2005.

Silva EV. Desenvolvimento de raízes finas em povoamentos monoespecíficos e mistos de Eucalyptus grandis e Acacia mangium [dissertação]. Piracicaba: Recursos Florestais, Universidade de São Paulo; 2007.

Vandermeer J. The Ecology of Intercropping. New York: Cambridge University Press; 1989.

Vezzani FM. Aspectos nutricionais de povoamentos puros e mistos de Eucalyptus saligna (Smith) e Acacia mearnsii (De Wild.) [dissertação]. Porto Alegre: Ciência do Solo, Universidade Federal do Rio Grande do Sul; 1997. PMid:8978741.

Vezzani FM, Tedesco MJ, Barros NF. Alterações dos nutrientes no solo e nas plantas em consórcio de eucalipto e acácia-negra. Revista Brasileira de Ciência do Solo 2001; 25(1): 225-231.

Viera M. Crescimento inicial e produtividade de plantios monoespecíficos e mistos de Eucalyptus urograndis e Acacia mearnsii em sistema agrossilvicultural [dissertação]. Santa Maria: Engenharia Florestal, Universidade Federal de Santa Maria; 2010.

Viera M, Schumacher MV, Liberalesso E. Crescimento e produtividade de povoamentos monoespecíficos e mistos de eucalipto e acácia-negra. Pesquisa Agropecuária Tropical 2011; 41(3): 415-421. http:// dx.doi.org/10.5216/pat.v41i3.10760

Viera M, Schumacher MV. Biomassa em povoamentos monoespecíficos e mistos de eucalipto e acácianegra e do milho em sistema agrossilvicultural. Cerne 2011; 17(2): 259-265. 